\title{
Vólvulo primario de íleon: una rara forma de obstrucción intestinal en el adulto. Reporte de caso y revisión de la literatura
}

\author{
Nelson López-Casillas ${ }^{1}$, Aarón Cuevas-González ${ }^{1}$ y Rodolfo Zambrano-Lechuga ${ }^{1}$
}

Primary volvulus of ileum: A rare form of intestinal obstruction in the adult. Case report and literature review

Background: The small bowel volvulus is caused by the abnormal rotation of the small intestine around the axis of its own mesentery. This can lead to intestinal obstruction, ischemia, infarction or perforation. Clinical case: A 71-year-old male patient with an acute abdominal pain, suspicious for a bowel occlusion, performed a surgical exploration, finding small bowel volvulus at the ileocecal valve level, with necrosis and ischemia of $280 \mathrm{~cm}$ of the small intestine, resulting in intestinal resection and terminal ileostomy. Still preserving $320 \mathrm{~cm}$ of viable small intestine from the duodenojejunal angle, with a satisfactory evolution. Discussion: Small bowel volvulus is an uncommon entity, and a life-threatening surgical emergency, that should be suspected in all patients with abrupt abdominal pain and signs of bowel obstruction, without previous abdominal surgery or other obvious causes. Early diagnosis and immediate surgical intervention are key factors associated with a better prognosis for this group of patients.

Key words: intestinal volvulus; intestinal obstruction; intestinal ischemia.

\section{Resumen}

Antecedentes: El vólvulo de intestino delgado se produce por el giro anormal del intestino delgado alrededor del eje de su propio mesenterio, lo cual puede generar obstrucción intestinal, isquemia, infarto o perforación. Caso clínico: Paciente masculino de 71 años que cursó con abdomen agudo. Sospechando cuadro de oclusión intestinal, se realizó exploración quirúrgica en la que se encontró como hallazgos vólvulo de intestino delgado en la válvula ileocecal, con isquemia y necrosis de $280 \mathrm{~cm}$ de intestino delgado, por lo cual se realizó resección intestinal e ileostomía terminal, preservando $320 \mathrm{~cm}$ de intestino delgado viable desde ángulo duodeno-yeyunal. Cursó con una evolución satisfactoria. Discusión: El vólvulo de intestino delgado es una entidad infrecuente y una urgencia quirúrgica que amenaza la vida. Se debe sospechar en todos los pacientes que presenten dolor abdominal abrupto y signos de obstrucción intestinal, sin cirugía abdominal previa ni otras causas obvias. El diagnóstico precoz y la intervención quirúrgica inmediata son factores clave asociados con un mejor pronóstico para este grupo de pacientes.

Palabras clave: vólvulo de intestino delgado; obstrucción intestinal; isquemia intestinal.

\section{Introducción}

El vólvulo de intestino delgado (VID) es una rara entidad clínica que representa alrededor del 1\% de todos los casos de oclusión intestinal en el adulto y su presentación clínica es la de abdomen agudo ${ }^{1}$. La mayoría de los casos serán diagnosticados en recién nacidos porque 1:500 nacidos vivos tendrá malrotación intestinal y aproximadamente el $80 \%$ de estos pacientes presentará VID en el primer mes de vida ${ }^{2-4}$.

Los tipos e incidencia de vólvulo intestinal presentan diferencias de acuerdo con la edad y la geografía. En adultos, el vólvulo de colon es relativamente común; en colon sigmoides se presenta en el $70-80 \%$ de los casos y en ciego en el $10-20 \%$ de los casos. En contraste, el VID es relativamente raro ${ }^{5}$.

Estudios realizados durante muchas décadas
'Servicio de Cirugía General, Hospital General Regional N. ${ }^{\circ} 1$, Instituto Mexicano del Seguro Social, Ciudad Obregón, Sonora, México.

Recibido el 22 de enero de 2017, aceptado para publicación el 28 de marzo de 2017.

Correspondencia a: Nelson López-Casillas drnelsonlc@live.com 
hasta la actualidad señalan que la incidencia anual es de 1,7-5,7 casos por 100.000 adultos en América y Norte de Europa y de 24-60 casos por 100.000 adultos en África, India, Medio Oriente y Asia ${ }^{6-8}$.

En 1909, Spasokukozkis reportó la primera serie de 28 de casos de vólvulo intestinal de la cual se tiene registro en la actualidad 9 .

Dependiendo de su etiología, podemos dividir el VID en primario y secundario ${ }^{10}$. El vólvulo primario ocurre en cavidades abdominales en las que no existe un defecto anatómico predisponente. Esto se presenta con mayor frecuencia en África, India y países de Medio Oriente. Estas altas incidencias parecen estar relacionadas con los hábitos alimentarios: en estas zonas es común la ingesta de grandes volúmenes de alimentos con alto contenido de fibra, después de períodos de ayunos prolongados durante el Ramadán ${ }^{11,12}$.

Por otra parte, el VID secundario ocurre en presencia de lesiones predisponentes, ya sean congénitas o adquiridas; estas incluyen: malrotaciones, bridas y adherencias, entre otras ${ }^{13}$. Es importante señalar que el VID no es sinónimo de malrotación intestinal, ya que no hay alteraciones anatómicas en la fijación intestinal y mesentérica.

Según un estudio retrospectivo realizado entre 1998 y 2010, el promedio de edad en la cual se presenta dicha entidad en el adulto fue $66,0 \pm 19,4$ años. Se presenta más comúnmente en el género femenino en una relación de 1,8-1 respecto a los hombres, según las últimas series de casos reportados por Welch ${ }^{2}$, Ruiz Tovar ${ }^{14}$ y Coe $^{15}$.

Actualmente no existen lineamientos que establezcan cuál es el tratamiento de elección, aunque el manejo quirúrgico siempre está indicado.

\section{Caso clínico}

Presentamos el caso de un hombre de 71 años de edad, sin antecedentes personales patológicos de importancia para el padecimiento. Con hábito tabáquico y alcohólico intensos, ambos desde los 15 años. Negaba cirugías previas u otros antecedentes relevantes.

Inicia su padecimiento 2 días previos al ingreso hospitalario con dolor abdominal de tipo cólico generalizado intenso (9/10), acompañado de náuseas, vómitos en 2 ocasiones, de contenido gastrobiliar, hiporexia, así como de evacuaciones disminuidas en consistencia en 6 ocasiones sin moco ni sangre, motivo por el cual acude al Servicio de Urgencias, donde recibió manejo a base de opioides.

Signos vitales: TA 133/73, FC 88, FR 19x', temperatura $36,5^{\circ} \mathrm{C}$. A la exploración física se ob- serva a un varón, de edad aparente a la cronológica, orientado en persona y espacio, cooperador, con piel y tegumentos con palidez ligera. Normocéfalo, con reflejos pupilares normales, cavidad oral con mucosa subhidratada, sonda nasogástrica con nulo gasto, cuello cilíndrico sin adenomegalias, con tráquea central, campos pulmonares con buena entrada y salida de aire, ruidos cardiacos con buen tono e intensidad, rítmicos, no ausculto agregados. Abdomen plano, depresible, peristalsis ausente, con muy ligero dolor a la palpación superficial y profunda en todo el marco cólico, sin datos de irritación peritoneal, sin megalias o masas palpables; extremidades íntegras, sin edema, con buen llenado capilar.

Los estudios de laboratorio reportaron una hemoglobina de 14,32 g/dl, hematocrito de 43,9\%, leucocitos de $8.700 / \mathrm{mm}^{3}$, neutrófilos de $71,1 \%$, linfocitos de $16,9 \%$, monocitos de $9,3 \%$, plaquetas de 191.000 , glucosa de 121 , creatinina de 0,98 , urea de 38,5 , bilirrubina total de 0,8 , bilirrubina directa de 0,2 , bilirrubina indirecta de 0,6 , amilasa de 130 , DHL de 134, Na de 135, K de 3,8, Cl de 107, TP 13,1 y TTP de 33,4.

Se realizaron radiografías de tórax AP, sin evidencia de aire libre subdiafragmático, ni derrames ni otras alteraciones. En las radiografías simples de abdomen se observa imagen en pila de monedas en el decúbito (Figura 1), así como asas de intestino delgado dilatadas, con niveles hidroáereos y zona

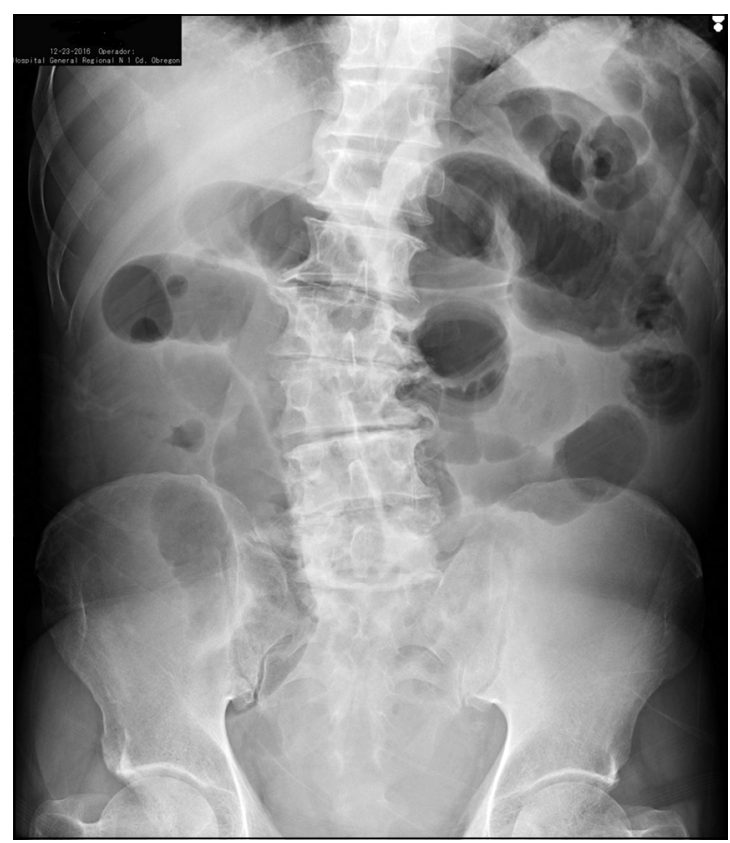

Figura 1. Radiografía abdominal decúbito que muestra imagen en pila de monedas. 


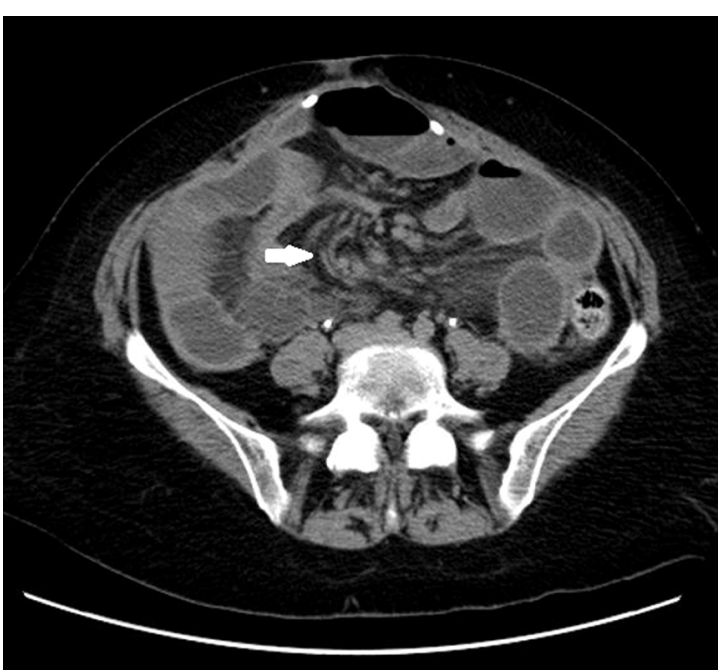

Figura 2. Signo del remolino, por rotación de intestino delgado alrededor de sus vasos mesentéricos.

transicional con ausencia de gas en colon y ámpula rectal en la radiografía de pie.

Además, se realizó ecografía abdominal, la cual reportó líquido libre en espacio supra e inframesocólico e interasa, dilatación importante de asas intestinales, con abundante contenido fecal, así como aperistalsis.

Ante la sospecha de un cuadro de oclusión intestinal, recibió manejo conservador mediante sonda nasogástrica descompresiva, ayuno y reposición hidroelectrolítica a requerimentos, así como analgésicos opioides durante $48 \mathrm{~h}$, con persistencia de los datos clínicos y radiográficos de oclusión intestinal. Se realizó tomografía abdominal en la que se encontró el signo del remolino, que indica la presencia de vólvulo intestinal (Figuras 2 y 3 ).

Se decidió realizar laparotomía exploradora, en la cual los hallazgos operatorios fueron los siguientes: VID a nivel de válvula ileocecal, con compromiso vascular irreversible de $280 \mathrm{~cm}$ de intestino delgado (Figura 4), por lo cual se realizó resección intestinal e ileostomía terminal, preservando $320 \mathrm{~cm}$ de intestino delgado viable desde el ángulo duodenoyeyunal. El examen de anatomía patológica evidenció signos de isquemia y necrosis en el intestino resecado, sin otras alteraciones de importancia.

Durante su período postoperatorio recibió manejo médico con dieta baja en fibra, loperamida (12 $\mathrm{mg}$ diarios fraccionados en 3 tomas), dieta en quintos para ostomizado, analgésicos parenterales y antibióticos de amplio espectro. Presentó adecuada mejoría clínica y fue egresado al $5 .^{\circ}$ día postoperatorio con un gasto por ileostomía menor a $500 \mathrm{cc}$ diarios.

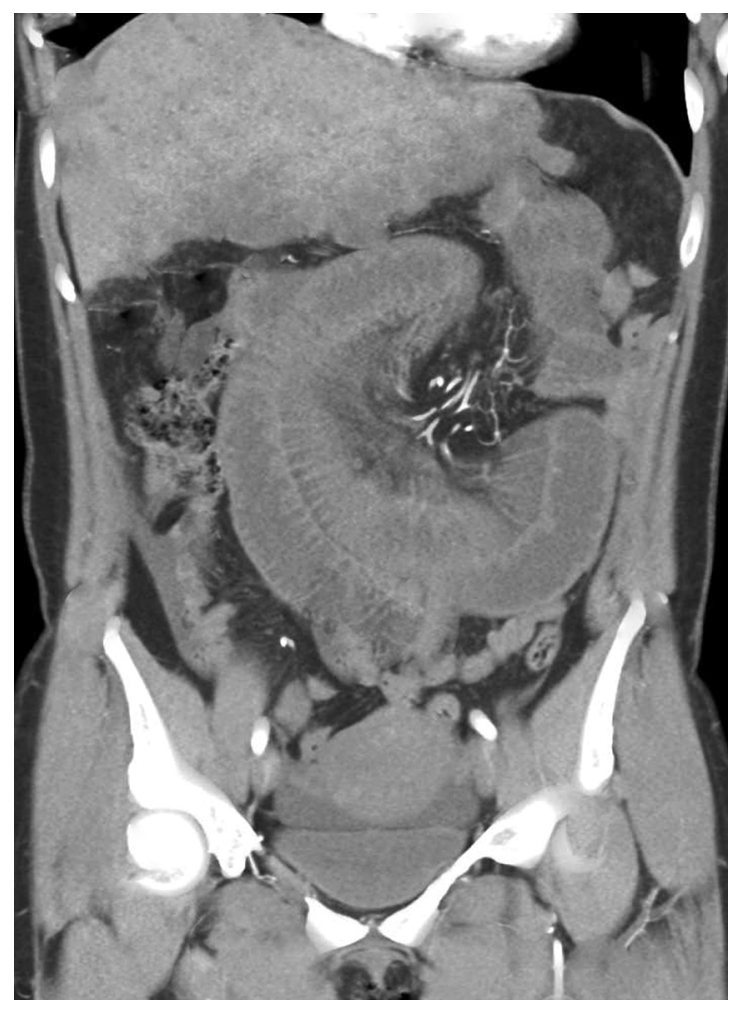

Figura 3. TC abdominopélvica: corte coronal.

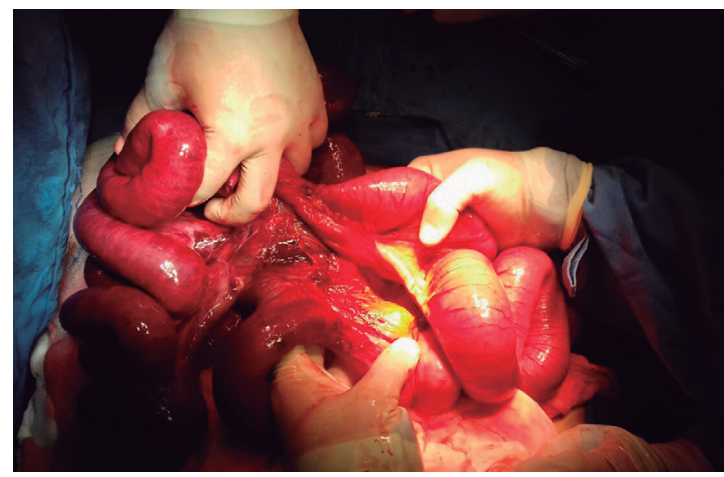

Figura 4. Intervención quirúrgica en la que se observa intestino delgado volvulado e isquémico a nivel de válvula ileocecal (a mano izquierda del cirujano) e íleon proximal viable (a mano derecha del cirujano).

\section{Discusión}

El termino vólvulo es derivado del latín 'volvere', que significa vuelta o giro. El VID se define como el giro anormal del intestino delgado alrededor del eje de su propio mesenterio. Esto puede producir obstrucción intestinal, isquemia, infarto o perforación ${ }^{16}$.

El VID se divide en primario y secundario. El vólvulo primario ocurre usualmente en niños y adultos jóvenes en los cuales no se encuentran anormalidades predisponentes durante la laparotomía, es raro en adultos mayores. 
Existen teorías para la presentación de VID primaria, como la teoría dietética propuesta por Spasokukozki ${ }^{9}$ que habla de que el vólvulo primario es causado por una combinación de factores: hipermovilidad, hipermotilidad, así como llenado rápido y repentino de un intestino vacío con grandes cantidades de alimento poco digerible que favorecen la rotación. Dicho autor describió detalladamente un mecanismo, con especial énfasis en la fuerte presión ejercida por las enérgicas ondas peristálticas en la dirección de menor resistencia: cuando se toman masas voluminosas de alimentos de escasa digestibilidad, las asas del yeyuno se dilatan y llenan $\mathrm{y}$ no encuentran resistencia del intestino delgado inferior, vacío, por lo que caen a la pelvis. Las asas vacías de la parte inferior de yeyuno e íleon son empujadas hacia arriba en una dirección en sentido horario que provoca una torsión de $180^{\circ}$. Cuando se vacía el estómago, el intestino es empujado hacia el cuadrante superior izquierdo, mientras que el bolo es transportado hacia adelante rellenando las asas distales, las cuales se vuelven pesadas y caen hacia abajo produciendo una rotación completa del mesenterio en sentido horario ${ }^{10}$.

Otra es la teoría anatómica, la cual refiere que, en las poblaciones de alto riesgo, el mesenterio correspondiente al intestino delgado es más largo, con una inserción más estrecha y una falta de grasa mesentérica ${ }^{10}$. Por otro lado, el vólvulo secundario del intestino medio en el que hay una lesión preexistente aparece habitualmente en los pacientes de más edad (de sexta a octava décadas de vida) ${ }^{14}$. La causa más frecuente es la adhesión postoperatoria. Sin embargo, se han reportado muchas otras causas en informes o series de casos, incluyendo hernias internas, tumores, ganglios mesentéricos, divertículo de Meckel, lipoma mesentérico, linfangioma mesentérico, embarazo, endometriosis, abscesos, micobacterias, aneurismas y hematomas, y ascaris ${ }^{17-24}$.

La presentación clínica del vólvulo primario del intestino medio suele ser inespecífica. Un comienzo abrupto de signos y síntomas de obstrucción del intestino delgado en un paciente sin cirugía abdominal previa ni otras causas evidentes (hernias), precedido por dolor cólico epigástrico o periumbilical varios días antes debería suscitar la sospecha de esta entidad. Más importante aún, el dolor fuera de proporción del grado de obstrucción observado en la isquemia mesentérica aguda y signos de respuesta inflamatoria sistémica (taquicardia, fiebre, taquipnea y leucocitosis) o peritonitis deben inducir al cirujano a operar urgentemente al paciente, debido al compromiso vascular intestinal ${ }^{16}$. El dolor abdominal es el síntoma más común en el 94-100\% de los pacien- tes y llega a ser de gran intensidad. La severidad del dolor está directamente relacionada con el grado de oclusión vascular y no con el grado de obstrucción intestinal, por lo que la intensidad del dolor no corresponde a los hallazgos clínicos, ya que solo el $26 \%$ de los pacientes tienen datos de irritación peritoneal ${ }^{17,25}$. También se pueden presentar náuseas en el $85 \%$, ser de inicio agudo en el $75 \%$, con distensión abdominal en el $55 \%$ de los pacientes ${ }^{17}$. Los hallazgos de laboratorio no son sensibles ni específicos para el diagnóstico de esta entidad: solo el $66 \%$ de los pacientes se presentan con torsión mesentérica y leucocitosis, aunque el $100 \%$ de los pacientes con necrosis intestinal tuvieron cuenta de leucocitos mayor a $10.000 / \mathrm{cm}^{3}$. Se reporta elevación de amilasa y lactato sérico, así como acidosis metabólica en el 55-75\% de los pacientes con VID con isquemia y necrosis, respectivamente, a diferencia de la elevación que solo aparece en el $4-5 \%$ de los pacientes sin sufrimiento de asa ${ }^{26,27}$.

La exploración diagnóstica preoperatoria incluye las radiografías de abdomen de pie y decúbito, la ecografía doppler a color, la tomografía computarizada abdominopélvica $\mathrm{y}$, más recientemente, la angiotomografía.

Las placas simples de abdomen son poco específicas para el diagnóstico de VID, pero tienen una gran sensibilidad para el diagnóstico de oclusión intestinal ${ }^{28}$. Pueden demostrar signos de obstrucción intestinal tales como niveles hidroaéreos y asas intestinales dilatadas, así como signos de isquemia intestinal o necrosis, tales como huellas dactilares y neumatosis intestinal o, en casos extremos, gas de vena porta. Los estudios baritados pueden llegar a ser útiles en ciertos casos, con el signo de Corskcrew o patrón en espiral ${ }^{29}$.

Se ha informado que la ecografía doppler a color es útil en el diagnóstico del VID, al identificar el giro de las asas intestinales y la vena mesentérica superior alrededor de la arteria mesentérica superior, que se denomina signo del remolino (whirlpool sign), con un $92 \%$ de sensibilidad y un $100 \%$ de especificidad $^{30-32}$. Las desventajas son el hecho de que es operador dependiente y que la interposición de gas puede limitar su sensibilidad.

La tomografía abdominal con contraste intravenoso es el método diagnóstico de elección: es rápida, no invasiva y tiene una sensibilidad que varía entre el 89 y el $100 \%{ }^{33}$. En dicho estudio se pueden observar datos poco específicos compatibles con obstrucción intestinal y signos característicos de la torsión mesentérica, como son el signo en espiral o el de remolino, también conocido como peacock's sign. Estos datos tomográficos se observan porque 
el intestino gira alrededor de los vasos mesentéricos, lo que genera esta clásica imagen ${ }^{34}$. La apariencia angiográfica de los vasos mesentéricos retorcidos, denominada signo del poste de peluquero, es patognomónica para vólvulo de intestino medio ${ }^{35}$. La angiotomografía ha sido introducida y ampliamente aceptada: produce imágenes tridimensionales multiplanares y proporciona información sobre la presencia del vólvulo, el grado y ubicación de la obstrucción intestinal, la presencia de isquemia intestinal y cualquier anomalía asociada de los órganos adyacentes ${ }^{36}$.

El vólvulo clínicamente asumido y radiológicamente demostrado requiere intervención quirúrgica inmediata debido al riesgo asociado de isquemia intestinal. La desvolvulación del intestino involucrado con frecuencia es la única maniobra que hay que realizar, aunque algunos autores recomiendan la fijación intestinal o incluso la resección para evitar la recurrencia del vólvulo ${ }^{8,14,37}$. A casi la mitad de los pacientes se les hará una resección de un intestino delgado gangrenoso ${ }^{33}$. En la actualidad, existen varios informes que describen el manejo laparoscópico del VID, considerando los beneficios de una estancia hospitalaria postoperatoria más corta, la reducción de las complicaciones postoperatorias y, posiblemente, un menor riesgo de adherencias posoperatorias en comparación con el abordaje abierto ${ }^{38-40}$.

El resultado de los pacientes con VID es peor cuando hay un retraso en el diagnóstico (debido a su rareza, especialmente en los países occidentales), la participación de pacientes mayores con comorbilidades asociadas y el desarrollo de isquemia intestinal y necrosis. La mortalidad oscila entre el 9 y el $33 \%$ en los pacientes explorados por VID según la serie consultada y, aunque Roggo y Ottinger ${ }^{17}$ reportaron un $0 \%$ de mortalidad siempre y cuando el intestino se encuentre viable, la mortalidad puede incrementarse del 20 hasta el $100 \%$ cuando existe necrosis intestinal ${ }^{41,42}$. En nuestro caso fue realizada resección intestinal e ileostomía terminal debido a la isquemia intestinal irreversible presentada y al estado nutricional del paciente, por alto riesgo de fuga anastomótica.

\section{Conclusión}

Se debe sospechar el vólvulo primario del intestino delgado en todos los pacientes que presenten dolor abdominal abrupto y signos de obstrucción intestinal, sin cirugía abdominal previa ni otras causas obvias. Las radiografías simples no son específicas y la ecografía doppler a color es operador dependiente. La modalidad de imagen de elección es la tomografía computarizada y la angiotomografía, más recientemente, que puede demostrar el intestino delgado y el mesenterio rotados, y proporciona información simultánea para cualquier isquemia intestinal asociada. El diagnóstico precoz y la intervención quirúrgica inmediata son factores clave asociados con un mejor pronóstico para este grupo de pacientes.

El caso antes descrito corresponde a un vólvulo primario de íleon en un paciente de género masculino, de la octava década de la vida, con isquemia y necrosis de un gran segmento del intestino, sin factores predisponentes aparentes para esta enfermedad.

\section{Responsabilidades éticas}

Protección de personas y animales. Los autores declaran que para esta investigación no se han realizado experimentos en seres humanos ni en animales.

Confidencialidad de los datos. Los autores declaran que han seguido los protocolos de su centro de trabajo sobre la publicación de datos de pacientes.

Derecho a la privacidad y consentimiento informado. Los autores declaran que en este artículo no aparecen datos de pacientes.

\section{Conflicto de intereses}

Los autores declaran no tener ningún conflicto de intereses.

\section{Bibliografía}

1. Spiro HM. Motor abnormalities. Clinical gastroenterology. $3^{\text {rd }}$ edition. Nueva York: McMillan; 1983. p. 492-506. Chapter 22.

2. Welch GH, Anderson JR. Volvulus of the small intestine in adults. World J Surg. 1986;10:496-500
3. Strouse PJ. Disorders of intestinal rotation and fixation (malrotation). Pediatr Radiol. 2004;34:837-51 [PubMed: 15378215].

4. Torres AM, Ziegler MM. Malrotation of the intestine. World J Surg. 1993;17:32631.

5. Ellis H. Acute intestinal obstruction. En: Schwartz SI, Ellis H (editors).
Maingot's abdominal operations $9^{\text {th }}$ edition. Volume 1, Chapter 39. Norwalk, CT: Appleton \& Lange; 1989. pp 855-904.

6. Cathcart RS 3rd, et al. Surgical treatment of midgut nonrotation in the adult, patient. Surg Gynecol Obstet. 1981;152:207-10 [PubMed: 7209763]. 
7. Gulati SM, et al. Volvulus of the small intestine in India. Am J Surg. 1973;126:661-4.

8. Iwuagwu ODG. Small bowel volvulus. A review. J R Coll Surg Edinb. 1999;44:1505.

9. Spasokukozki S. Volvulus Intestinorum als Krankheit des hungermden Menschen. Arch Klin Chir. 1909;91:211.

10. Vaez-Zadeh K, Dutz W, Nowrooz-Zadeh $\mathrm{M}$. Volvulus of the small intestine in adults. A study of predisposing factors. Ann Surg. 1969;169:265-71.

11. Ford EG, Senac MO Jr, Srikanth MS, Weitman JJ. Malrotation of the intestine in children. Ann Surg. 1992;215:172-8.

12. Ghebrat K. Trend of small intestinal volvulus in north western Ethiopia. East Afr Med J. 1998;75:549-52.

13. Izes BA, Scholz FJ, Munson JL. Midgut volvulus in an elderly patient. Gastrointest Radiol. 1992;17:102-4.

14. Ruiz-Tovar J1, Morales V, Sanjuanbenito A, Lobo E, Martinez-Molina E. Volvulus of the small bowel in adults. Am Surg. 2009;75:1179-82.

15. Coe T, Chang D, Sicklick J. Small bowel volvulus in the adult populace of the United States: Results from a populationbased study. Am J Surg. 2015;210:20110.e2, http://dx.doi.org/10.1016/j. amjsurg.2014.12.048.

16. White RR, Jacobs DO. Volvulus of the stomach and small bowel. En: Charles Yeo, et al., editors. Shackelford's surgery of the alimentary tract., $1,6^{\text {th }}$ ed. Philadelphia: Elsevier Saunders; 2007. p. 1035-7.

17. Roggo A, Ottinger LW. Acute small bowel volvulus in adults. A sporadic form of strangulating intestinal obstruction. Ann Surg. 1992;216:135-41.

18. Catalano OA, Bencivenga A, Abbate M, Tomei E, Napolitano M, Vanzulli A. Internal hernia with volvulus and intussusception. Case report. Abdom Imaging. 2004;29:164-5.

19. Bissen L, Brasseur P, Sukkarich F, Takieddine M, Frecourt N. Jejunal lipomatosis with intussusception and volvulus. A case report. J Radiol. 2004;85:128-30.

20. Qayyum A, Cowling MG, Adam EJ. Small bowel volvulus related to a calcified mesenteric lymph node. Clin Radiol. 2000;55:483-5.

21. Sheen AJ, Drake I, George PP. A small bowel volvulus caused by a mesenteric lipoma. Report of a case. Surg Today. 2003;33:617-9.

22. Jang JH, Lee SL, Ku YM, An CH, Chang ED. Small bowel volvulus induced by mesenteric lymphangioma in an adult. A case report. Korean J Radiol. 2009;10:319-22.

23. Wax JR, Christie TL. Complete small bowel volvulus complicating the second trimester. Obstet Gynecol. 1993;82 Suppl:689-91.

24. Furukawa A, Yamasaki M, Furuichi K, Yokoyama K, Nagata T, Takahashi M, et al. Helical CT in the diagnosis of small bowel obstruction. Radiographics. 2001;21:341-55.

25. Fisher JK. Computed tomographic diagnosis of volvulus in intestinal malrotation. Radiology. 1981;140: 145-6.

26. Sarr MG, Bulkley GB, Zuidema GD. Preoperative recognition of intestinal strangulation obstruction: Prospective evaluation of diagnostic capability. Am J Surg. 1983;145:176-82.

27. Mucha P. Small intestinal obstruction. Surg Clin North Am. 1987;67:597-620.

28. Hochman DJ, Nivatvongs S, Selvasekar CR, Tantitemit T. The Noble plication. New indication for a historical procedure. Tech Coloproctol. 2007;11:152-4.

29. Bernestein SM, Russ PD. Midgut volvulus. A rare cause of acute abdomen in an adult patient. AJR Am J Roentgenol. 1998;171:639-41.

30. Duran C, Ozturk E, Uraz S, Kocakusak A, Mutlu H, Killi R. Midgut volvulus value of multidetector computed tomography in diagnosis. Turk J Gastroenterol. 2008;19:189-92.

31. Shimanuki Y, Aihara T, Takano H,
Moritani T, Oguma E, Kuroki H, et al. Clockwise whirlpool sign at color Doppler US an objective and definite sign of midgut volvulus. Radiology. 1996;199:261-4.

32. Pracros JP, Sann L, Genin G, Tran-Minh VA, Morin de Finfe CH, Foray P, et al. Ultrasound diagnosis of midgut volvulus the whirlpool sign. Pediatr Radiol. 1992;22:18-20.

33. Ho YC. Venous cut-off sign as an adjunct to the whirl sign in recognizing acute small bowel volvulus via CT scan. J Gastrointest Surg. 2012;16:2005-6.

34. Huang JC, Shin JS, Huang YT, Chao CJ, Ho SC, Wu MJ, et al. Small bowel volvulus among adults. J Gastroenterol Hepatol. 2005;20: 1906-12.

35. Buranasiri SI, Baum S, Nusbaum M, Tumen $\mathrm{H}$. The angiographic diagnosis of midgut malrotation with volvulus in adults. Radiology. 1973;109:555-6.

36. Feng ST, Chan T, Sun CH, Li ZP, Guo HY, Yang GQ, et al. Multiphasic MDCT in small bowel volvulus. Eur J Radiol. 2010;76:e13-8.

37. Kim KH, Kim MC, Kim SH, Park KJ, Jung GJ. Laparoscopic management of a primary small bowel volvulus: A case report. Surg Laparosc Endosc Percutan Tech. 2007;17:335-8.

38. Liauw JJ, Cheah WK. Laparoscopic management of acute small bowel obstruction. Asian J Surg. 2005;28:185-8.

39. Kirshtein B, Roy-Shapira A, Lantsberg L, Avinoach E, Mizrahi S. Laparoscopic management of acute small bowel obstruction. Surg Endosc. 2005;19:464-7.

40. Navez B, Arimont JM, Guiot P. Laparoscopic approach in acute small bowel obstruction. A review of 68 patients. Hepatogastroenterology. 1998;45:2146-50.

41. Frazee RC, Mucha P Jr, Farnell MB, van Heerden JA. Volvulus of the small intestine. Ann Surg. 1988;208:565-8.

42. Agrawal RL, Misra MK. Volvulus of the small intestine in Northern India. Am J Surg. 1970;120:366-70. 